\title{
CORRELAÇÕES FENOTÍPICA, GENÉTICA ADITIVA E AMBIENTAL EM CENOURA $\left({ }^{1}\right)$
}

\author{
WALTER JOSÉ SIQUEIRA $\left({ }^{2,6}\right)$, ROHLF DIETER ILLG $\left({ }^{3}\right)$, JOÃO BAPTISTA FORNASIER (4), \\ NEWTON PRADO GRANJA $\left({ }^{2}\right)$, ROGÉRIO SALES LISBÃO ( ${ }^{4}$ ) \\ e RUI RIBEIRO DOS SANTOS ( 5 )
}

\begin{abstract}
RESUMO
A obtenção de variedades comerciais envolve, por via de regra, a seleção de vários caracteres simultaneamente. Sendo assim, o conhecimento prévio de suas inter-relações também pode ser fundamental na estratégia a ser adotada pelo melhorista. $O$ objetivo do presente trabalho foi estimar as correlações fenotipica, genética aditiva e de ambiente para diversos pares de caracteres de interesse agronómico de cenoura (Daucus carota L.), variedade Campinas, nas épocas de outono-inverno e primavera-verão, na Estação Experimental de Monte Alegre do Sul (SP), do Instituto Agronômico de Campinas. Esse germoplasma, originário da Seção de Hortaliças do referido Instituto, apresenta elevado nivel de resistência à queima das folhas e grande variação quanto ao formato de raízes. Além disso, revela tendência para emissão precoce da haste floral após estímulos de baixa temperatura e/ou fotoperiodo longo, durante o estádio vegetativo da cultura. O delineamento foi de blocos ao acaso com 102 progênies de meios-irmãos e quatro e três repetições para as épocas de outono-inverno e primavera-verão respectivamente. As correlaçōes fenotípica ( $\mathrm{r}_{\overline{\mathrm{F}}}$ ), genética aditiva $\left(r_{A}\right)$ e de ambiente ( $\left.\mathrm{r}_{\mathrm{E}}\right)$ foram estimadas nas duas épocas de plantio, mediante análises de covariância entre pares de caracteres. As correlaçðes fenotípicas, de valores significativos, e as correlações genéticas aditivas, foram negativas somente para os pares de caracteres envolvendo a porcentagem de florescimento prematuro (FL) ou a porcentagem de raízes com defeito (DEF), sendo positivas para as demais associações de caracteres. As correlações genéticas aditivas foram de alta magnitude $(>0,5)$ para a maioria dos pares de caracteres analisados nas duas épocas de plantio. A seleção para a produção de raízes cilíndricas comerciáveis (CILC) mostra-se favorável em ambas as épocas de plantio, na redução do caráter FL, bem como no aumento de produção de raizes comerciáveis (PC) e produção total (PT). Finalmente, discute-se a participação relativa dos efeitos de ambiente e genéticos na correlação fenotipica.
\end{abstract}

Termos de indexação: cenoura, Daucus carota L., correlações, seleção.

( ${ }^{1}$ ) Parte de tese do primeiro autor, submetida à UNICAMP e defendida em 10/8/89 para obtenção do Título de Mestre em Ciências Biológicas, área de concentração Genética Vegetal. Recebido para publicação em 26 de janeiro e aceito em 30 de abril de 1993.

(2) Instituto Agronômico de Campinas (IAC), Caixa Postal 28, 13001-970 Campinas (SP).

$\left({ }^{3}\right)$ Departamento de Genética e Evolução, UNICAMP, Caixa Postal 6109, 13081-000 Campinas (SP).

(4) Instituto Agronômico (IAC). In memoriam.

(5) Estação Experimental de Monte Alegre do Sul (IAC).

( ${ }^{6}$ Com bolsa de pesquisa do CNPq. 


\section{ABSTRACT \\ PHENOTYPIC, ADDITIVE GENETIC AND ENVIRONMENTAL CORRELATIONS IN CARROTS}

Since development of commercial varieties involves simultaneous selection for various traits, previous knowledge of their interrelationships may also be fundamental to the breeder as the strategies to be adopted. The objective of this research was to estimate phenotypic, additive genetic and environmental correlations for a number of traits of agronomic interest in carrot (Daucus carota L.) cultivar Campinas, grown during the fall-winter and spring-summer seasons at the Experiment Station of Monte Alegre do Sul, Instituto Agronômico de Campinas (IAC), State of São Paulo, Brazil. This germplasm, originated from the "Seção de Hortaliças" (IAC), presents, besides a high level of resistance to Alternaria dauci, a large variation for root shape and a tendency to early bolting, stimulated by low temperatures and/or long photoperiods during the crop vegetative stage. Data were obtained by evaluating 102 half-sib progenies on a randomized block design experiment with four and three replications, respectively, for the fall-winter and spring-summer planting dates. Phenotypic ( $\mathrm{r}_{\mathrm{F}}$ ), additive genetic $\left(\mathrm{r}_{\mathrm{A}}\right)$ and environmental $\left(\mathrm{r}_{\mathrm{E}}\right)$ correlations were estimated by covariance analyses between pairs of characters, in both planting dates. The phenotypic and additive genetic correlations were negative only for pairs of characters involving percentage of early flowering (EF) or percentage of defective roots, while the correlations were high $(>0.5)$ for most of the pairs of characters in both planting dates. Selection for percentage of cylindric marketable roots will lead to reduction in EF as well as increases in marketable root yield and total root yield in both planting. This research further presents a discussion of the relative participation of environmental and genetic effects in the phenotypic correlation.

Index terms: carrot, Daucus carota L., correlations, selection.

\section{INTRODUÇÃO}

O cultivo tradicional de cenoura no Estado de São Paulo, bem como nas regiões Sudeste e Centro-Oeste do País, é realizado durante o período onde predominam baixas temperaturas, ou seja, no outono-inverno, empregando-se a variedade Nantes, de raízes com formato cilíndrico, originária da França e tida como padrão de qualidade pelos olericultores.

Em razão da existência de diversas seleções derivadas dessa variedade, Nantes passou também a ser considerada como um grupo. Da mesma maneira, as variedades recomendadas ao plantio sob temperaturas elevadas, ou seja, na primavera-verão, mas de raízes com formato cônico ou quase cilíndrico, com resistência à queima das folhas (Alternaria dauci) e sensibilidade à baixa temperatura para indução ao florescimento, pertencem a outro grupo, denominado Kuroda.
Num terceiro grupo (Nacional), estão as variedades ou populações originárias do Sul, que floresce, em taxas variadas, por estímulos de fotoperíodo longo ( $>12,5$ horas de luz) e crescente (Costa, 1974), tais como Brasília, Tropical, Campinas, Nacional e Londrinense.

As instituições públicas e privadas vêm-se empenhando na seleção dentro dessas populaçð̃es do terceiro grupo, visando, entre outros caracteres, à qualidade de raízes e à ausência de florescimento prematuro nos plantios antecipados, a partir de agosto, nas condições paulistas. Ressalte-se que as variedades do grupo Nacional também florescem prematura e variavelmente, quando submetidas às baixas temperaturas durante 0 plantio de outono-inverno. Sendo assim, pode-se também realizar a seleção no sentido de aprimorar essas populações para o plantio na época tradicional, uma vez que seu potencial produtivo tem-se mostrado superior à 'Nantes', apesar 
da qualidade inferior de suas raizes (Peixoto \& Della Vecchia, 1984; Faoro et al., 1985).

Existem fatores atuando contra ou a favor das forças seletivas, quando se deseja alterar as freqüências gênicas de determinada população. Segundo Paterniani \& Miranda Filho (1978), entre esses fatores podem ser mencionados a variabilidade genética presente na população original, que, por sua vez, resulta da freqūência gênica original; o método de seleção empregado; o tamanho efetivo da população; a técnica e a precisão das avaliações dos genótipos; a influência do ambiente; os efeitos pleiotrópicos e as correlações fenotípicas, genotípicàs e de ambiente.

Nesse aspecto, o estudo de correlações entre caracteres é muito importante do ponto de vista do melhoramento genético, pois, em geral, o aprimoramento de determinada população ou variedade é direcionado para um conjunto de caracteres simultaneamente (Vencovsky, 1978). Os coeficientes de correlação têm sido determinados em algumas hortaliças, no sentido de conhecer a natureza e a magnitude das associações entre caracteres para o fornecimento de subsídios ao melhorista quanto à estratégia a ser adotada durante a seleção (Buso, 1978; Candeia et al., 1986; Miranda et al., 1988). Algumas associações entre caracteres de cenoura têm sido constatadas por simples observações de dados experimentais, sem, contudo, terem sido realizados estudos pormenorizados de correlação. Guedes \& Nascimento (1985) observaram uma associação fenotípica positiva entre diâmetro de raiz $e$ produção de sementes em umbelas primárias e secundárias da variedade Brasília. Vieira \& Casali (1984) relataram que, quanto maior o vigor da parte aérea da cenoura, maior o diâmetro do xilema, que possui tendência à coloração mais clara do que o córtex ou floema da raiz. McCollum (1971) concluiu que o esverdeamento externo da raiz não possui correlação genética com peso, comprimento, diâmetro de raiz $\mathrm{e}$ índice de forma (comprimento/diâmetro) em três populações de cenoura. Por outro lado, houve correlação fenotípica para o esverdeamento ex- terno e interno, com valores ao redor de 0,75 . Desse modo, a seleção para coloração externa normal resultaria numa coloração interna sem esverdeamento. Observou-se, também, alta correlação genética e positiva para indice de forma com comprimento de raiz. Em 1980, Braghandani \& Choudhrury, citados por Vieira \& Casali (1984), mostraram que o diâmetro de raíz teve efeito direto pronunciado sobre a produção e que a altura de folhagem teve efeito positivo indireto sobre o diâmetro de raiz.

O objetivo do presente trabalho foi estimar, com vistas ao melhoramento genético, as correlações fenotípica, genética aditiva e de ambiente entre pares de caracteres de interesse agronômico da variedade Campinas, lançada pelo IAC em 1965, nos cultivos de outono-inverno e primavera-verão.

\section{MATERIAL E MÉTODOS}

Em janeiro de 1987, foram obtidas 102 progênies de meios-irmãos da variedade Campinas, em campo de multiplicação de sementes localizado na Estação Experimental de Monte Alegre do Sul (SP), do Instituto Agronômico. Essa variedade, apesar do elevado nível de resistência à queima das folhas (Camargo, 1984), vem apresentando grande variação fenotípica quanto ao formato de raízes e tendência para emissão precoce da haste floral após estímulos de baixas temperaturas no plantio de outono-inverno e/ou de fotoperíodo longo no plantio de primavera-verão. Portanto, a sensibilidade ao frio e ao fotoperiodo longo, causando o florescimento prematuro e a ausência de padrão definido de formato de raízes, não logrou o êxito dessa variedade junto aos olericultores. Dois experimentos foram instalados na mesma Estação Experimental, com semeaduras em abril e outubro de 1987 , correspondendo, na prática, ao cultivo denominado de outono-inverno e de primavera-verão respectivamente. Os canteiros, em ambas as épocas de plantio, foram preparados antecipadamente, realizando-se a correção do $\mathrm{pH}$ do solo pela saturação de bases trocáveis a $\mathrm{V}=80 \% \mathrm{e}$ 
adubação orgânica e mineral de acordo com Fornasier \& Lisbão (1987). A densidade de semeadura foi de, aproximadamente, 1,0 $\mathrm{g}$ de sementes por metro linear em sulcos transversais ao canteiro, espaçados de $25 \mathrm{~cm}$, sendo a área da parcela de $1 \mathrm{~m}^{2}$. O delineamento experimental empregado foi de blocos ao acaso com quatro e três repetições, respectivamente, para a primeira e para a segunda época de plantio.

Realizaram-se dois desbastes, deixando-se cerca de 60 plantas por parcela em ambos os experimentos. Os demais tratos culturais, como controle de ervas daninhas, irrigação, adubação de cobertura etc., foram realizados de acordo com as recomendações técnicas próprias à cultura da cenoura.

Os caracteres avaliados em porcentagem foram calculados em relação ao número total de plantas da parcela, sendo indicados a seguir: (a) florescimento prematuro (FL), expresso pelo número de plantas com haste floral durante o ciclo vegetativo; (b) raízes cônicas comerciáveis (CONC), definidas como o número de raízes sem defeito, de formato cônico e comprimento mínimo de $7 \mathrm{~cm}$; (c) raízes cilindricas comerciáveis (CILC), definidas como o número de raízes sem defeito, de formato cilíndrico e comprimento mínimo de $7 \mathrm{~cm}$; (d) raízes cilíndricas comerciáveis de extremidade arredondada (CILCR); (e) raizes cilíndricas comerciáveis de extremidade afilada (CILCA) e (f) raízes com defeito (DEF), definidas como o número de raízes apresentando defeitos como rachaduras, ombro verde, bifurcações, radicelas.

Foram ainda avaliados e expressos em grama/ /parcela os caracteres: (a) peso médio de raízes cônicas comerciáveis (PMCONC), obtido a partir do coeficiente peso/número de raízes cônicas comerciáveis; (b) peso médio de raizes cilíndricas comerciáveis (PMCILC), definido pela razão entre peso/número de raízes cilíndricas comerciáveis; (c) produção comerciável (PC), obtida a partir do somatório dos pesos de raízes cônicas e cilíndricas comerciáveis, e (d) produção total (PT), que inclui o peso de raízes de plantas florescidas e de raízes com defeito.
Na segunda época de plantio (primavera-verão), coletaram-se os dados para as mesmas características, excetuando-se CILCR e CILCA. Os dados de porcentagem obtidos nas duas épocas de plantio foram transformados para arco seno $\sqrt{\%}$, como sugerido por Sokal \& Rohlf (1969). Os valores de 0 e $100 \%$ foram substituidos, respectivamente, por $0,25 / \mathrm{n}$ e $100-(0,25 / \mathrm{n})$, sendo $n$ igual ao número de raízes por parcela (Steel \& Torrie, 1960). Os demais caracteres foram analisados nas unidades originais em que foram coletados.

Estudaram-se os seguintes pares de caracteres no primeiro experimento: $F L \times$ (PT, PC, DEF, CILC, CILCR, CILCA, CONC), CONC x (PT, PC, DEF) e CILC x (PT, PC, DEF). No segundo, analisaram-se os pares de caracteres mencionados, com exceção de CILCR e CILCA.

O modelo matemático, correspondendo ao delineamento de blocos ao acaso para os dois experimentos, considerando-se todos os componentes (exceto a média) de efeito aleatório é mostrado a seguir:

$$
Y_{i j}=m+t_{i}+b_{j}+e_{i j}
$$

onde:

m: média geral; $t_{i}$ : efeito do tratamento $i$; $b_{j}$ : efeito do bloco $\mathrm{j}$; $\mathrm{e}_{\mathrm{ij}}$ : efeito do erro experimental associado ao tratamento $\mathrm{i}$ do bloco $\mathrm{j}$.

As estimativas das variâncias e covariâncias dos componentes das esperanças matemáticas foram obtidas mediante manipulação dos quadrados médios e produtos médios das análises da variância e covariância respectivamente, e os coeficientes de correlação genética aditiva $\left(r_{A}\right)$, fenotípica entre médias de progênies $(r F)$ e de ambiente ( $\left.r_{E}\right)$ foram estimados para cada época de plantio como segue (Vencovsky, 1978):

$$
\begin{aligned}
& r_{A x y}=\operatorname{CoV} p_{x y} / \sqrt{\sigma^{2} p_{x} \cdot \sigma^{2} p_{y}} \\
& r_{F x y}=\operatorname{PMp}_{x y} / \sqrt{Q M p_{x} \cdot Q M p_{y}} e \\
& r_{E x y}=\mathrm{PMr}_{x y} / \sqrt{\mathrm{QMr}_{x} \cdot \mathrm{QMr}_{y}}
\end{aligned}
$$


Nessas expressões, COVpxy refere-se à covariância genética de progênies entre as características $\mathrm{X}$ e $\mathrm{Y} ; \sigma^{2} \mathrm{p}_{\mathrm{x}}$ e $\sigma^{2} \mathrm{p}_{\mathrm{y}}$, às variâncias genéticas de progênies dos caracteres $\mathrm{X}$ e $\mathrm{Y}$ respectivamente, $\mathrm{PM}_{\mathrm{p}}$ e $\mathrm{PM}_{\mathrm{r}}$, aos produtos médios de progênies e de resíduos, e $\mathbf{Q M}_{\mathrm{p}}$ e $\mathrm{QM}_{\mathbf{r}}$, aos quadrados médios de progênies e resíduos respectivamente.

A fim de verificar, em termos relativos, a contribuiçåo dos efeitos genéticos aditivos vs. demais efeitos na correlação fenotípica entre os pares de caracteres avaliados, utilizou-se - desdobramento da expressão de $\mathrm{r}_{\mathrm{F}}$, segundo Falconer (1981):

$$
\begin{aligned}
\mathrm{r}_{\mathrm{F} x y} & =\sqrt{h^{2} \cdot h^{2} y} \cdot r_{A x y}+ \\
& +\sqrt{\left(1-h^{2}\right)\left(1-h^{2} y\right)} \cdot r_{E x y} .
\end{aligned}
$$

O primeiro termo da fórmula refere-se aos efeitos genéticos aditivos (G) e o segundo corresponde aos demais efeitos, aqui denominados simplificadamente de ambiental (E).

Sendo assim, as relações $(\mathrm{G} / \mathrm{r} \overline{\mathrm{F}}) .100 \mathrm{e}$ $(E / r \bar{F}) .100$ mostram a contribuição relativa (\%) dos dois tipos de contribuição na correlação fenotípica, independente do seu sinal. A herdabilidade no sentido restrito para médias de progênies de meios-irmãos foi obtida de acordo com a expressão apresentada por Vencovsky (1978).

\section{RESULTADOS E DISCUSSÃO}

Os resultados das correlações entre pares de caracteres da primeira e da segunda época são mostrados, respectivamente, nos quadros 1 e 2. Todas as associações envolvendo os caracteres FL ou DEF com os demais foram negativas ( $\mathrm{T} \overline{\mathrm{F}}$, $r_{A}$ e $\left.r_{E}\right)$, exceto para FL $x$ PT e CONC $x$ DEF na segunda época de plantio, respectivamente, com $\mathrm{r}_{\overrightarrow{\mathrm{F}}}=0,0038$ e $\mathrm{r}_{\mathrm{A}}=0,0412$ de sinais positivos, porém 'de valores próximos de zero. Portanto, à medida que se selecionam para caracteres de formato ou peso de raízes, espera-se, por conseqüência, na maioria dos casos, decréscimos no florescimento prematuro e nas raízes com defeito.
As correlações genéticas aditivas mostraram-se similares em magnitude em relação às fenotípicas, nas duas épocas de plantio, evidenciando forte correspondência da seleção baseada no fenótipo com os efeitos genéticos dos pares de caracteres analisados. $O$ caráter FL mostrou correlação genética aditiva de alta magnitude e de sinal negativo com $\mathrm{PC}\left(\mathrm{r}_{\mathrm{A}}=-0,9117\right)$ e de baixa magnitude para PT $\left(r_{A}=-0,3912\right)$; portanto, a seleção para PC deverá causar reduções no FL. Por outro lado, na segunda época de plantio, a produção total também é negativamente correlacionada, mas de elevada magnitude em termos aditivos com o FL ( $\left.r_{A}=-0,8682\right)$, mostrando estreita associação entre o florescimento prematuro e o peso total. Como o caráter PC é mais influenciado diretamente pelo número de plantas florescidas na parcela, pois o DEF foi de baixa frequêencia, esperam-se aumentos substanciais no peso de raízes comerciáveis com a gradual eliminação do FL em ambas as épocas de plantio. Essa associação se caracteriza por elevadas estimativas de correlação genética aditiva ( $r_{A}=-0,9117$ na primeira época e $r_{A}$ $=-0,9490$ na segunda).

Como o PC reúne os pesos obtidos dos dois formatos comerciáveis (cônicas + cilindricas), a seleção direta para CILC, que é o caráter mais desejável no melhoramento, é mais indicada, uma vez que o nivel de correlação genética aditiva FL $x$ CILC apresentou também alta magnitude $\left(r_{\mathrm{A}}=-0,6556\right.$ para a primeira época $\mathrm{e} \mathrm{r}_{\mathrm{A}}=$ $-0,7257$ para a segunda). A seleção voltada para CILC, em vez de CONC, mostra-se, também, mais favorável no aumento de PT e PC no outono-inverno, e pouco menos favorável na primavera-verão. Os caracteres CONC x DEF e CILC $x$ DEF nas duas épocas de plantio se caracterizaram pela baixa magnitude de $r_{A} e$, portanto, forte influência nos demais efeitos não controlados no experimento, que ocorrem dentro de progênies. A seleção dos caracteres derivados de CILC, avaliados somente na primeira época de plantio, indica maiores vantagens para CILCA na redução de $F L$ do que CILCR, em razão da maior magnitude de $r_{A}$ obtida, ou seja, $-0,7748$ e $-0,5924$ respectivamente. 
Quadro 1. Estimativas dos coeficientes de correlação fenotípica ( $\left.\mathrm{r}_{\overrightarrow{\mathrm{F}}}\right)$, genética aditiva ( $\mathrm{r}_{\mathrm{A}}$ ) e de ambiente $\left(\mathrm{r}_{\mathrm{E}}\right)$ de pares de caracteres avaliados ao nível de parcelas, da época de plantio de outono-inverno

\begin{tabular}{|c|c|c|c|}
\hline Caracteres $\left({ }^{1}\right)$ & $\mathbf{r}_{\mathrm{A}}$ & $\Gamma_{\bar{F}}\left({ }^{2}\right)$ & $r_{E}$ \\
\hline FL $x$ PT & $-0,3912 \pm 0,0920$ & $-0,3551 * * \pm 0,0935$ & $-0,2905 \pm 0,0550$ \\
\hline FL $\times$ PC & $-0,9117 \pm 0,0411$ & $-0,8815^{* *} \pm 0,0472$ & $-0,6844 \pm 0,0418$ \\
\hline FL $\times$ DEF & $-0,6228 \pm 0,0782$ & $-0,5758^{* *} \pm 0,0818$ & $-0,4749 \pm 0,0506$ \\
\hline FL $x$ CILC & $-0,6556 \pm 0,0755$ & $-0,6091 * * \pm 0,0793$ & $-0,3574 \pm 0,0537$ \\
\hline FL $\times$ CILCR & $-0,5924 \pm 0,0806$ & $-0,5403^{* *} \pm 0,0841$ & $-0,3400 \pm 0,0540$ \\
\hline FL $\times$ CILCA & $-0,7748 \pm 0,0632$ & $-0,7142 * * \pm 0,0700$ & $-0,5035 \pm 0,0496$ \\
\hline FL $\times$ CONC & $-0,6382 \pm 0,0770$ & $-0,6076 * \pm 0,0794$ & $-0,5072 \pm 0,0495$ \\
\hline CONC $\times$ PT & $0,1253 \pm 0,0992$ & $0,0466 \mathrm{~ns} \pm 0,0999$ & $-0,1559 \pm 0,0567$ \\
\hline CONC $\times \mathrm{PC}$ & $0,2932 \pm 0,0956$ & $0,2695^{* *} \pm 0,0963$ & $0,1739 \pm 0,0566$ \\
\hline CONC $\times$ DEF & $-0,1829 \pm 0,0983$ & $-0,2363 * \pm 0,0972$ & $-0,3895 \pm 0,0529$ \\
\hline CILC $\times$ PT & $0,6059 \pm 0,0796$ & $0,4503 * * \pm 0,0893$ & $0,0178 \pm 0,0574$ \\
\hline CILC $\times$ PC & $0,6554 \pm 0,0755$ & $0,6109 * * \pm 0,0792$ & $0,3958 \pm 0,0528$ \\
\hline CILC $\times$ DEF & $-0,2368 \pm 0,0972$ & $-0,2863^{* *} \pm 0,0958$ & $-0,4517 \pm 0,0512$ \\
\hline
\end{tabular}

(') FL: \% florescimento prematuro; CILC: \% de raizes cilindricas comerciáveis; CILCR: \% raizes cilindricas comerciáveis de extremidade arredondada; CILCA: \% de raizes cilindricas comerciáveis de extremidade afilada; CONC: \% de raizes cônicas comerciáveis; DEF: \% de raízes com defeitos; PT: produção total de raizes, e PC: produção comerciável de raízes.

$\left(^{2}\right)^{* *}=$ significativo a $1 \%$ pelo teste $t ;{ }^{*}=$ significativo a $5 \%$ pelo teste $t ; n s=$ não significativo.

Quadro 2. Estimativas dos coeficientes de correlação fenotípica ( $\left.\mathrm{r}_{\bar{F}}\right)$, genética aditiva ( $\left.\mathrm{r}_{\mathrm{A}}\right)$ e de ambiente $\left(r_{E}\right)$ de pares de caracteres avaliados ao nível de parcelas, da época de plantio de primavera-verão

Caracteres $\left({ }^{1}\right)$

$\mathbf{r}_{\mathrm{A}} \quad \mathrm{r}_{\mathbf{F}}\left(^{2}\right)$

$r_{E}$

\begin{tabular}{lrrr} 
FL $\times$ PT & $-0,8682 \pm 0,0496$ & $-0,7493^{* *} \pm 0,0663$ & $0,0038 \pm 0,0703$ \\
FL $\times$ PC & $-0,9490 \pm 0,0315$ & $-0,9167^{* *} \pm 0,0400$ & $-0,6557 \pm 0,0532$ \\
FL $\times$ DEF & $-0,5564 \pm 0,0831$ & $-0,4911^{* *} \pm 0,0871$ & $-0,4979 \pm 0,0610$ \\
FL $\times$ CILC & $-0,7257 \pm 0,0688$ & $-0,6625^{* *} \pm 0,0749$ & $-0,4511 \pm 0,0628$ \\
FL $\times$ CONC & $-0,8683 \pm 0,0496$ & $-0,8167^{* *} \pm 0,0577$ & $-0,5230 \pm 0,0600$ \\
CONC x PT & $0,7963 \pm 0,0605$ & $0,6604^{* *} \pm 0,0751$ & $0,0721 \pm 0,0702$ \\
CONC x PC & $0,8980 \pm 0,0440$ & $0,8404^{* *} \pm 0,0542$ & $0,5542 \pm 0,0586$ \\
CONC x DEF & $0,0412 \pm 0,0999$ & $-0,0930 \mathrm{~ns} \pm 0,0996$ & $-0,4206 \pm 0,0638$ \\
CILC $\times$ PT & $0,8010 \pm 0,0599$ & $0,6404^{* *} \pm 0,0768$ & $0,1214 \pm 0,0698$ \\
CILC $\times$ PC & $0,8290 \pm 0,0559$ & $0,7286^{* *} \pm 0,0685$ & $0,3868 \pm 0,0649$ \\
CILC $\times$ DEF & $-0,1593 \pm 0,0987$ & $-0,2714^{* *} \pm 0,0962$ & $-0,4905 \pm 0,0613$ \\
\hline
\end{tabular}

(1) FL: \% florescimento prematuro; CILC: \% de raízes cilíndricas comerciáveis; CONC: \% de raízes cônicas comerciáveis; DEF: \% de raízes com defeitos; PT: produçăo total de raízes e PC: produção comerciável de raízes.

$\left({ }^{2}\right)^{* *}=$ significativo a $1 \%$ pelo teste $t ; *=$ significativo a $5 \%$ pelo teste $t ; n s=$ nåo significativo. 
Os resultados da correlação ambiental, $\mathrm{r}_{\mathrm{E}}$, são discutidos a seguir, embora, pelo tipo de progênie utilizado, essa correlação também inclua as variaçб̃es genéticas existentes dentro de progênies e, como não são controladas experimentalmente, incidem, portanto, no erro experimental.

Pelos resultados do quadro 1 , referentes à época de outono-inverno, a correlação ambiental apresentou valores relativamente altos para os pares de caracteres FL $x$ PC $(-0,6844), F L x$ DEF $(-0,4749)$, FL $x$ CILCA $(-0,5035)$, FL $x$ CONC $(-0,5072)$ e CILC $x$ DEF $(-0,4517)$.

Entre as correlações de ambiente da primeira época de plantio, observa-se que $25,0 \%$ ficaram acima de 0,$5 ; 50,0 \%$ entre 0,3 e 0,5 , e $25,0 \%$ abaixo de 0,3 . Na segunda época de plantio, a magnitude das correlações de ambiente foi, em geral, semelhante à época anterior (Quadro 2). O maior valor de TE observado foi para FL $x$ PC (rE $=-0,6557)$. Cerca de $35,0 \%$ de rE ficaram acima de 0,5 e os demais $65,0 \%$, entre 0,5 e 0,3 . Como muitas das correlações re, obtidas nas duas épocas de plantio, foram de elevada magnitude, constituindo, portanto, fontes adicionais e incontroláveis de interferência nas associações dos caracteres, procurou-se quantificar, em termos relativos, a contribuição geral desses efeitos, na correlação fenotípica. Isso foi possível mediante o emprego da fórmula de $\mathrm{r} \overline{\mathrm{F}}$ apresentada por Falconer (1981) e indicada em Material e Métodos. Na fórmula, utiliza-se a herdabilidade obtida para cada caráter ( $X$ e Y), cujos valores obtidos nas duas épocas de plantio são mostrados no quadro 3 .

Os resultados sobre a participação ou contribuição relativa dos efeitos genéticos aditivos (G\%) e dos outros efeitos (E\%) na correlação fenotípica de valor significativo da primeira época de plantio, encontram-se no quadro 4.

Neste, observa-se a superioridade da contribuição relativa dos efeitos genéticos aditivos na correlação fenotípica, em comparação com os demais efeitos. Os efeitos de ambiente em FL $x$ CONC, CILC, CILCR, CILCA, DEF, PT e PC, são baixos, apresentando o máximo de $14,17 \%$ de contribuição para $\mathrm{rF}$. Portanto, na correlação fenotípica envolvendo o caráter FL, predominam os efeitos genéticos de natureza aditiva liberados entre progênies. Para os caracteres CONC x PC e CONC x DEF, os valores observados de $\mathrm{E} \%$ foram, respectivamente, 11,98 e $56,91 \%$, sendo este último o único da primeira época de plantio onde houve maior influência dos efeitos presentes no erro experimental na correlação fenotípica.

Quadro 3. Estimativas dos coeficientes de herdabilidade $\left(h^{2}\right)$ nas duas épocas de plantio

$\begin{array}{lll}\text { Caracteres }\left({ }^{1}\right) & h^{2} \text { (outono-inverno) } & h^{2} \text { (primavera-verão) }\end{array}$

$\%$

FL

90,76

91,48

CONC

77,09

91,43

CILC

80,84

73,39

CILCR

74,93

CILCA

74,41

70,16

$-$

DEF

85,08

54,56

PC

66,74

87,76

PT

81,97

(1) FL: \% florescimento prematuro; CILC: \% de raízes cilíndricas comerciáveis; CILCR: \% de raízes cilindricas comerciáveis de extremidade arredondada; CILCA: \% de raizes cilindricas comerciáveis de extremidade afilada; CONC: \% de raizes cônicas comerciáveis; DEF: \% de raizes com defeitos; PT: produção total de raizes e PC: produção comerciável de raízes. 
Quadro 4. Contribuição relativa de efeitos genéticos aditivos (G\%) vs. demais efeitos genéticos mais ambiente $(E \%)$ na correlação fenotípica $(r \bar{F})$ de pares de caracteres avaliados ao nível de parcelas (outono-inverno)

Caracteres $\left({ }^{1}\right)$

$\mathbf{G}$

E

$\mathrm{r} \overline{\mathrm{F}}$

\begin{tabular}{|c|c|c|c|c|}
\hline & & & - & \\
\hline \multirow[t]{7}{*}{ FL } & vs. $\mathrm{CONC}$ & 87,86 & 12,14 & $-0,6076$ \\
\hline & vs. CILC & 92,19 & 7,81 & $-0,6091$ \\
\hline & vs. CILCR & 90,42 & 9,58 & $-0,5403$ \\
\hline & vs. CILCA & 89,15 & 10,85 & $-0,7142$ \\
\hline & vs. DEF & 86,31 & 13,69 & $-0,5758$ \\
\hline & vs. PT & 85,83 & 14,17 & $-0,3551$ \\
\hline & vs. $\mathrm{PC}$ & 91,00 & 9,00 & $-0,8815$ \\
\hline \multirow[t]{2}{*}{ CONC } & vs. DEF & 43,09 & 56,91 & $-0,2363$ \\
\hline & vs. $\mathrm{PC}$ & 88,02 & 11,98 & 0,2695 \\
\hline \multirow[t]{3}{*}{ CILC } & vs. DEF & 62,28 & 37,72 & $-0,2863$ \\
\hline & vs. PT & 99,01 & 0,99 & 0,4503 \\
\hline & vs. $\mathrm{PC}$ & 89,13 & 10,87 & 0,6109 \\
\hline
\end{tabular}

(') Apenas para correlą̧ões fenotípicas que apresentaram significância pelo teste t ( 5 ou 1\%). FL: \% florescimento prematuro; CILC: \% de raizes cilindricas comerciáveis; CILCR: \% de raizes cilindricas comerciáveis de extremidade arredondada; CILCA: \% de raizes cilindricas comerciáveis de extremidade afilada; CONC: \% de raizes cônicas comerciáveis; DEF: \% de raízes com defeitos; PT: produção total de raízes e PC: produção comerciável de raízes.

As combinações CILC $x$ PT e CILC $x$ PC apresentaram, também, predominância de associações dos efeitos genéticos aditivos na composição final da correlação fenotípica, ou seja, respectivamente, de 99,01 e $89,13 \%$. A maior magnitude da correlação ambiental de CILC x DEF $(-0,4517)$ resultou em $E$ de $37,72 \%$, que só não foi maior em razão dos elevados niveis de herdabilidade obtidos para CILC $(80,84 \%)$ e DEF $(70,16 \%)$ (Quadro 3).

Quanto à segunda época de plantio, referente ao ciclo de primavera-verão, a contribuição de $\mathrm{E} \%$ na correlação fenotípica de valor significativo entre os caracteres avaliados, com exceção de CILC x DEF $(E \%=62,85)$, foi também baixa, não excedendo $20,0 \%$ (Quadro 5). Os maiores efeitos de natureza genética aditiva (G\%), nas associações dos caracteres, foram observados para FL $x$ PT $(\simeq 100,0 \%)$, FL $x$ PC $(92,76 \%)$, FL $x$ CONC $(92,05 \%)$, CONC x PT $(98,05 \%)$, CILC x PT $(95,69 \%)$ e CILC $x$ PC $(90,50 \%)$. Os demais pares de caracteres

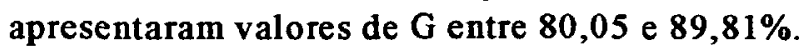
O valor obtido para a contribuição de $\mathrm{E} \%$ foi quase o dobro de G\% na correlação fenotípica de CILC $x$ DEF, evidenciando a dificuldade na redução de raízes defeituosas, a partir da seleção de CILC nas condiçðes de primavera-verão. Deve-se salientar, no entanto, que as freqüências de defeitos na população, em ambas as épocas đe plantio, foram baixas, não excedendo, suas médias, $8,0 \%$. Portanto, apesar de as correlações de ambiente terem sido altas para alguns pares de caracteres, nos dois experimentos, a influência para $r \bar{F}$ foi pequena na maioria dos casos, em razão das elevadas estimativas de herdabilidade observadas. 
Quadro 5. Contribuição relativa de efeitos genéticos aditivos (G\%) vs. demais efeitos genéticos mais ambiente $(\mathrm{E} \%)$ na correlação fenotípica $(\bar{r} \overline{\mathrm{F}})$ de pares de caracteres avaliados ao nível de parcelas (primavera-verão)

Caracteres $\left(^{1}\right)$

G

$\mathbf{E}$

$\mathbf{\Gamma} \overline{\mathbf{F}}$

\begin{tabular}{|c|c|c|c|c|}
\hline & & & & \\
\hline \multirow[t]{5}{*}{ FL } & vs. $\mathrm{PT}$ & $\simeq 100,00$ & 0,00 & $-0,7483$ \\
\hline & vs. $\quad \mathrm{PC}$ & 92,76 & 7,24 & $-0,9167$ \\
\hline & vs. $C O N C$ & 92,05 & 7,95 & $-0,8167$ \\
\hline & vs. CILC & 89,75 & 10,25 & $-0,6625$ \\
\hline & vs. DEF & 80,05 & 19,95 & $-0,4911$ \\
\hline \multirow[t]{2}{*}{ CONC } & vs. PT & 98,05 & 1,95 & 0,6604 \\
\hline & vs. $\quad P C$ & 89,81 & 10,19 & 0,8404 \\
\hline \multirow[t]{3}{*}{ CILC } & vs. $\mathrm{PT}$ & 95,69 & 4,31 & 0,6404 \\
\hline & vs. $\quad P C$ & 90,50 & 9,50 & 0,7286 \\
\hline & vs. DEF & 37,15 & 62,85 & $-0,2714$ \\
\hline
\end{tabular}

( $\left.{ }^{1}\right)$ Apenas para correlaçðes fenotípicas que apresentaram significância pelo teste 1 ( 5 ou 1\%). FL: \% florescimento prematuro; CILC: \% de raizes cilíndricas comerciáveis; CONC: \% de raízes cônicas comerciáveis; DEF: \% de raizes com defeitos; PT: produção total de raizes e PC: produção comerciável de raízes.

\section{CONCLUSÕES}

1. Houve predominância de efeitos genéticos aditivos na correlação fenotípica, para todos os pares de caracteres estudados, à exceção de: raízes com defeitos $\mathrm{x}$ raízes cônicas comerciáveis da primeira época e raízes com defeitos $x$ raizes cilínđricas comerciáveis da segunda época de plantio.

2. As correlações genéticas aditivas foram de alta magnitude $(>0,5)$ para a maioria dos pares de caracteres analisados em ambas as épocas de plantio.

3. Pelos níveis das correlações fenotípica e genética aditiva observados, a seleção para raízes cilíndricas comerciáveis mostra-se favorável na redução do florescimento prematuro e aumento do peso comerciável de raízes, com ligeira vantagem para as condições experimentais da segunda época de plantio.

\section{AGRADECIMENTOS}

Os autores agradecem ao Técnico Agrícola Roberto Faria a colaboração na condução dos experimentos e avaliaçðes de campo.

\section{REFERÊNCIAS BIBLIOGRÁFICAS}

BUSO, J.A. Estimativa de parâmetros genéticos de caracteres de planta e bulbo de cebola (Allium cepa $L$.). Piracicaba, 1978. 132p. Dissertação (Mestrado) ESALQ-USP, 1978.

CAMARGO, L. de S. As hortaliças e seu cultivo. 2.ed. Campinas, Fundação Cargill, 1984. 448p.

CANDEIA, J.A.; SILVA, N. da \& ZANOTO, M.D. Parâmetros genéticos e correlaçðes em cebola "Pira tropical". Horticultura Brasileira, Brasília, 4(2):17-19, 1986.

COSTA, C.P. da. Cenoura Nacional, um germoplasma para as condições de dias curtos nas regiōes tropicais e subtropicais. Relatório Cientifico do Instituto de Genética, Piracicaba, (8):50-52, 1974. 
FALCONER, D.S. Introdução à genética quantitativa. Viçosa, Universidade Federal de Viçosa, 1981. 279p.

FAORO, I.D.; BECKER, W.F. \& BUSATO, M.V. Comportamento de cultivares de cenoura na semeadura de setembro e outubro, em Caçador, SC. Horticultura Brasileira, Brasilia, 3(1):68, 1985.

FORNASIER, J.B. \& LISBÃO, R.S. Cenoura. In: INSTITUTO AGRONÔMICO (Campinas). Instruçzes agrtcolas para o Estado de Sao Paulo. 4.ed. Campinas, 1987. p.63-64. (Boletim, 200)

GUEDES, A.C. \& NASCIMENTO, W.M. Influência do diâmetro da raiz sobre a produção de sementes de cenoura (Daucus carota L.) cv. Brasilia. Horticultura Brasileira, Brasilia, 3(1):73, 1985.

McCOLLUM, G.O. Greening of carrot roots (Daucus carota L.): Estimates of heritability and correlation. Euphytica, Wageningen, 20:549-560, 1971.

MIRANDA, J.E.C. de.; COSTA, C.P. da \& CRUZ, C.D. Correlações genotipica, fenotípica e de ambiente entre caracteres de fruto e planta de pimentão (Capsicum annuum L.). Revista Brasileira de Genética, Ribeirão Preto, 11(2):457-468, 1988.
PATERNIANI, E. \& MIRANDA FILHO, J.B. Melhoramento de populações. In: PATERNIANI, E., coord. Melhoramento e produção de milho no Brasil. Campinas, Fundação Cargill, 1978. cap.6, p.202-256.

PEIXOTO, N. \& DELLA VECCHIA, P.T. Avalią̧ão de cultivares de cenoura em Goiânia. In: CONGRESSO BRASILEIRO DE OLERICULTURA, 24., e REUNIÃO LATINO-AMERICANA DE OLERICULTURA, 1., Jaboticabal, 1984. Resumos. Jaboticabal, UNESP-FCAV, 1984. p.30.

SOKAL, R.R. \& ROHLF, F.J. Biometry: the principles and practice of statistics in biological research. San Francisco, W.H. Freeman, 1969. 776p.

STEEL, R.G.D. \& TORRIE, J.H. Principles and procedures of statistics. New York, McGraw Hill, 1960. $481 \mathrm{p}$.

VENCOVSKY, R. Herança quantitativa. In: PATERNIANI, E., coord. Melhoramento e produção de milho no Brasil. Campinas, Fundação Cargill, 1978. cap.5, p.122-201.

VIEIRA, J.V. \& CASALI, V.W.D. Melhoramento da cenoura para verão. Informe Agropecuário, Belo Horizonte, 10(120):17-18, 1984. 